\title{
NEW APPROACH TO FOLDING WITH THE COULOMB WAVE FUNCTION
}

Blokhintsev L.D. ${ }^{1}$, Kadyrov A.S. ${ }^{2}$, Mukhamedzhanov A.M. ${ }^{3}$, Savin D.A. ${ }^{1}$

${ }^{1}$ Skobeltsyn Institute of Nuclear Physics, Lomonosov Moscow State University, Russia;

${ }^{2}$ Curtin University, Perth, Australia; ${ }^{3}$ Cyclotron Institute, Texas A\&M University, College Station, USA

E-mail: blokh@srd.sinp.msu.ru

A promising way to account for the Coulomb interaction in integral equations of the quantum few-body problem is the transition from the free Green function to the Coulomb one. To this end one should fold kernels of the integral equations with the Coulomb wave functions $[1,2]$. This is a rather intricate and time consuming computing problem.

In the present work a new approach to folding a function with the Coulomb wave is proposed. The method is based on expanding the partial-wave Coulomb scattering function in the configuration space in a series of spherical Bessel functions. This allows one to significantly simplify the calculations. To test the method, a simple trial function is chosen for which the folding with the Coulomb scattering wave function can be calculated analytically. Such a choice allows us to estimate the accuracy of the expansion used. Specifically, we choose a partial-wave component of the simple pole propagator proportional to the Legendre function of the second kind $Q_{l}$, with $l$ being the orbital angular momentum. In the following table the relative difference between the exact value of the folding and the value obtained by replacing the Coulomb partial wave function by a finite number of terms of the series is presented for different values of $l$ and the Coulomb parameter $\eta$.

\begin{tabular}{|c|c|c|c|c|c|c|c|c|c|c|c|}
\hline$l$ & $\eta$ & \multicolumn{8}{|c|}{ Number of terms } \\
\cline { 3 - 10 } & & 1 & 2 & 3 & 4 & 5 & 6 & 7 & 8 & 9 & 10 \\
\hline 0 & 0.231 & $5 \cdot 10^{-}$ & $2 \cdot 10^{-}$ & $2 \cdot 10^{-}$ & $1 \cdot 10^{-}$ & $6 \cdot 10^{-}$ & $2 \cdot 10^{-}$ & $2 \cdot 10^{-}$ & $3 \cdot 10^{-}$ & $6 \cdot 10^{-11}$ & $2 \cdot 10^{-12}$ \\
\hline 1 & 0.231 & $5 \cdot 10^{-}$ & $2 \cdot 10^{-}$ & $3 \cdot 10^{-}$ & $2 \cdot 10^{-}$ & $1 \cdot 10^{-}$ & $5 \cdot 10^{-}$ & $2 \cdot 10^{-}$ & $4 \cdot 10^{-}$ & $3 \cdot 10^{-10}$ & $5 \cdot 10^{-11}$ \\
\hline 2 & 0.231 & $5 \cdot 10^{-}$ & $2 \cdot 10^{-}$ & $4 \cdot 10^{-}$ & $3 \cdot 10^{-}$ & $3 \cdot 10^{-}$ & $2 \cdot 10^{-}$ & $2 \cdot 10^{-}$ & $2 \cdot 10^{-}$ & $4 \cdot 10^{-10}$ & $8 \cdot 10^{-11}$ \\
\hline 0 & 2.31 & $5 \cdot 10^{-}$ & $1 \cdot 10^{-}$ & $2 \cdot 10^{-}$ & $2 \cdot 10^{-}$ & $4 \cdot 10^{-}$ & $1 \cdot 10^{-}$ & $7 \cdot 10^{-}$ & $6 \cdot 10^{-}$ & $1 \cdot 10^{-}$ & $1 \cdot 10^{-}$ \\
\hline
\end{tabular}

The values 0.231 and 2.31 chosen for $\eta$ correspond to the proton-deuteron scattering at the center-of-mass energy of $300 \mathrm{keV}$ and $3 \mathrm{keV}$, respectively. One can see that for $\eta=0.231$ the two first terms of the series result in the accuracy within 0.002 . For $\eta=2.31$ the convergence is slower, however, even for this rather large value of the Coulomb parameter it is sufficient to take into account the first four terms in order to obtain the accuracy within 0.002 . The obtained results allow us to conclude that the proposed method is promising in solving the integral equations for quantum systems of few charged particles.

1. A.M.Mukhamedzhanov, V.Eremenko, A.I.Sattarov // Phys. Rev. C. 2012. V.86. 034001 .

2. E.I.Dolinsky, A.M.Mukhamedzhanov // Yad. Fiz. 1966. V.3. P.252. 